\title{
Avaliação neuropsicológica dos transtornos psicológicos na infância: um estudo de revisão
}

\author{
Jeane Lessinger Borges - Universidade Federal do Rio Grande do Sul1 \\ Clarissa Marceli Trentini - Universidade Federal do Rio Grande do Sul \\ Denise Ruschel Bandeira - Universidade Federal do Rio Grande do Sul \\ Débora Dalbosco Dell'Aglio - Universidade Federal do Rio Grande do Sul
}

\begin{abstract}
Resumo
Este artigo apresenta uma revisão da literatura sobre a avaliação neuropsicológica dos déficits cognitivos associados aos transtornos psicológicos na infância e adolescência, com base em pesquisas publicadas no período de 2000 a 2006. Foi realizado um levantamento abrangendo publicações nacionais e internacionais indexadas nas bases de dados Medline, SciELo e PsycInfo. Os resultados indicaram um aumento da produção científica na área da avaliação neuropsicológica do transtorno de déficit de atenção e hiperatividade, autismo, transtornos de humor e transtorno de conduta. Observou-se a presença de poucos estudos nacionais, indicando a necessidade de pesquisas na área de avaliação neuropsicológica no Brasil.

Palavras-chave: Avaliação neuropsicológica; Transtornos psicológicos; Testes; Infância.
\end{abstract}

\section{Neuropsychological assessment of psychological disorders in childhood: A review study}

\begin{abstract}
This study comprises a review about neuropsychological assessment of cognitive deficits associated to psychological disorders in childhood and adolescence, including researches published between 2000 and 2006. A survey of national and international publications indexed on Medline, SciELo and PsychoInfo was carried out. The results showed an increase of scientific publications within the neuropsychological assessment area of the Attentiondeficit/hyperactivity disorder, autism, mood disorders and conduct disorder. A lack of national studies was observed, indicating the necessity of more research in the neuropsychological assessment in Brazil.

Keywords: Neuropsychological assessment; Psychological disorders; Tests; Childhood.
\end{abstract}

\section{Introdução}

A avaliação neuropsicológica tem como objetivo estudar as relações entre a atividade cerebral, cognição e o comportamento (Lezak, Howieson \& Loring, 2004). Esse tipo de avaliação se baseia na análise funcional dos processos cognitivos (linguagem, memória, percepção, visocontrução, funções executivas) e na compreensão multidimensional dos prejuízos cognitivos. Assim, compreende-se que as alterações cognitivas, comportamentais e emocionais variam de acordo com a natureza, extensão e localização da lesão cerebral, bem como são influenciadas pelas variáveis idade, gênero, condições físicas e contexto psicossocial de desenvolvimento (Lezak e colaboradores, 2004).

A avaliação neuropsicológica da criança e adolescente tem sido comumente realizada nos casos de problemas neurológicos (Anderson, Anderson, Northam, Jacobs \& Mikiewicz, 2002; Steinlin e colaboradores, 2003).
Dessa forma, a importância da avaliação neuropsicológica de crianças foi destacada na investigação de dano cerebral após traumatismo craniano e acidente vascular (Donders \& Nesbit-Greene, 2004; Guimarães, Ciasca \& MouraRibeiro, 2002), na avaliação pré e pós-intervenção cirúrgica (Guimarães, Souza, Montenegro, Cendes \& Guerreiro, 2003) e em problemas de aprendizagem (Lindsay, Tomazic, Levine \& Accardo, 2001).

De forma mais restrita, o uso da avaliação neuropsicológica para os quadros psicopatológicos da infância e adolescência tem sido descrito apenas recentemente. Pesquisas sobre avaliação das funções cognitivas associadas ao transtorno de déficit de atenção e hiperatividade (Koschack, Kunert, Derichs, Weniger \& Irle, 2003; Rubia e colaboradores, 2001), ao transtorno de estresse pós-traumático (Beers \& De Bellis, 2002), aos transtornos de ansiedade (Toren e colaboradores, 2000), ao transtorno obsessivo-compulsivo (Roth, Milovan, Baribeau \& O'Connor, 2005) e aos transtornos de humor

\footnotetext{
${ }^{1}$ Endereço para correspondência:

Universidade Federal do Rio Grande do Sul - Núcleo de Estudos e Pesquisas em Adolescência (NEPA/UFRGS)

Rua Ramiro Barcelos, 2.600 - sala 115 - Santa Cecília - 90035-003 - Porto Alegre-RS

E-mail: jelessinger@ig.com.br
} 
têm sido realizadas (Cataldo, Nobile, Lorusso, Battaglia \& Molteni, 2005).

De um modo geral, essas pesquisas demonstram alterações no funcionamento dos sistemas cerebrais em relação aos transtornos mentais na infância e na adolescência, contribuindo para uma melhor compreensão teórica sobre as bases neurobiológicas subjacentes a esses quadros. Contudo, parece haver uma lacuna na literatura brasileira no que se refere a este tema. Assim, considerando a importância da avaliação neuropsicológica infantil para a investigação clínica dos transtornos psicológicos, este estudo apresenta uma revisão da literatura sobre o tema e descreve brevemente os instrumentos neuropsicológicos mais utilizados nesta área.

\section{Método}

Foi realizada uma revisão de literatura investigando os estudos teóricos e empíricos relacionados à avaliação neuropsicológica dos transtornos psicológicos em crianças e adolescentes nos seguintes Bancos de Dados: Medline, SciELo e PsycInfo. "Os descritores utilizados foram os seguintes termos: "avaliação neuropsicológica", "testes neuropsicológicos", "neuropsychological evaluation", "neuropsychological assessment" e "neuropychological battery", tendo sido selecionada a faixa etária de zero a 14 anos. Neste estudo, foram selecionados artigos publicados em revistas científicas nacionais e internacionais no período de janeiro de 2000 a junho de 2006. Inicialmente, foram encontrados 1.919 artigos. Buscou-se, então, refinar esta primeira seleção, buscando dar uma maior ênfase aos estudos sobre o uso da avaliação neuropsicológica dos transtornos psicológicos na infância e adolescência. $O$ título e o resumo dos artigos foram utilizados nesta segunda etapa e, ao final, 154 artigos foram selecionados para esta revisão. A partir desse resultado, uma análise de freqüência da avaliação neuropsicológica e o tipo de transtorno psicológico foi realizada.

\section{Resultados}

A Tabela 1 apresenta a freqüência de artigos sobre os prejuízos neuropsicológicos associados a diferentes transtornos psicológicos em crianças e adolescentes, por transtorno e por ano. De um modo geral, observou-se que há uma crescente produção científica referente ao TDAH $(65,60 \%)$. Ainda foram encontrados estudos sobre a avaliação neuropsicológica do autismo $(16,80 \%)$, dos transtornos de humor $(9,74 \%)$ e transtorno de conduta $(7,80 \%)$ em crianças e adolescentes.

Tabela 1 - Descrição da freqüência de artigos por transtorno e por ano na avaliação neuropsicológica infantil

\begin{tabular}{|c|c|c|c|c|c|c|c|c|c|}
\hline Transtorno psicológico/ano & 2000 & 2001 & 2002 & 2003 & 2004 & 2005 & 2006 & $\mathrm{n}$ & $\%$ \\
\hline Avaliação neuropsicológica do TDAH & 10 & 17 & 19 & 8 & 18 & 29 & & 101 & 65,60 \\
\hline Avaliação neuropsicológica do autismo & 6 & 5 & 2 & & 2 & 7 & 4 & 26 & 16,80 \\
\hline $\begin{array}{l}\text { Avaliação neuropsicológica dos } \\
\text { Transtornos de humor }\end{array}$ & & 1 & 2 & & 5 & 7 & & 15 & 9,74 \\
\hline $\begin{array}{l}\text { Avaliação neuropsicológica do transtorno } \\
\text { de conduta }\end{array}$ & 3 & & 3 & & 4 & 2 & & 12 & 7,80 \\
\hline Total & 19 & 23 & 26 & 8 & 29 & 45 & 4 & 154 & 100 \\
\hline
\end{tabular}

Transtorno de déficit de atenção e hiperatividade (TDAH)

Para a descrição sobre as pesquisas na área da avaliação neuropsicológica do TDAH foram selecionados 26 estudos, dos 101 encontrados nesta revisão, os quais têm ressaltado a importância do papel das funções executivas (FE) e do córtex frontal neste transtorno. Ressalta-se que foram encontrados dois estudos nacionais. No primeiro, Amaral e Guerreiro (2001) apresentaram uma proposta de avaliação neuropsicológica para o diagnóstico de TDAH. No segundo, Schmitz e colaboradores (2002) sugeriram diferenças no desempenho cognitivo entre adolescentes com diferentes subtipos de TDAH, indicando a necessidade de diagnóstico específico.

Os artigos revisados revelaram uma associação entre prejuízos nas FE e o TDAH (Reeve \& Schandler,
2001; Shallice e colaboradores, 2002). Crianças com TDAH têm apresentado baixo desempenho na inibição de resposta, no planejamento de tarefas e na atenção seletiva, e um maior aumento na perseveração de resposta, quando comparadas a grupos controle (Papadopoulos, Panayiotou, Spanoudis \& Natsopoulos, 2005; Schulz e colaboradores, 2005). Pesquisas indicaram diferentes direções de desfecho no desempenho cognitivo quando são avaliadas as variáveis (a) subtipos do TDAH: desatento, hiperativo e misto (Hinshaw, Sam, Treuting, Carte \& Zupan, 2002; Nigg, Blaskey, Huan-Pollock \& Rappley, 2002; Schmitz e colaboradores, 2002); (b) gênero (Seidman e colaboradores, 2005); (c) período do desenvolvimento (Drechsler, Brandeis, Foldenyi, Imhof \& Steinhausen, 2005); e (d) presença de comorbidade psiquiátrica (Fischer, Barkley, Smallish \& Fletcher, 2005; Oosterlaan, Scheres \& Sergeant, Psico-USF, v. 13, n. 1, p. 125-133, jan./jun. 2008 
2005; Sarkis, Sarkis, Marshall \& Archer, 2005). Ainda foram citados déficits na memória imediata como um importante componente neuropsicológico do TDAH (Cornoldi e colaboradores, 2001; Westerberg, Hirvikoski, Forssberg \& Klingberg, 2004).

Estudos investigaram o uso do metilfenidato em crianças com TDAH, apontando os benefícios deste na atenção, memória imediata e tarefas visoespaciais (Bedard, Ickowicz \& Tannock, 2002; Bedard, Martinussen, Ickowicz \& Tannock, 2004; O'Driscoll e colaboradores, 2005). Na mesma direção, os resultados indicaram que o efeito do Metilfenidato interfere de forma diferenciada na atenção, dependendo da dose utilizada (Konrad, Günther, Hanisch \& Herpertz-Dahlmann, 2004).

Os artigos revisados apontaram uma maior validade preditiva e discriminante para o diagnóstico de TDAH e seus déficits cognitivos quando se utilizam múltiplos instrumentos neuropsicológicos para avaliação de FE (Doyle, Biederman, Seidam, Weber \& Faraone, 2000; Perugini, Harvey, Lovejoy, Sandstrom \& Webb, 2000). Um estudo de metanálise descreveu os principais instrumentos neuropsicológicos utilizados para a avaliação do TDAH, entre eles, o Continuous Performance Test (CPT), Gordon Diagnostic System (GDS), Children's Checking Task (CCT), Stop Signal Task, Trail Making Test, Wisconsin Card Sorting Test (WCST), Stroop Interference Task, entre outros (Frazier, Demaree \& Youngstrom, 2004).

Particularmente, dois instrumentos foram mais utilizados na avaliação cognitiva do TDAH nas pesquisas revisadas: o Wisconsin Card Sorting Test (WCST) (ou Teste Wisconsin de Classificação de Cartas) e o Stroop Color-Word Test. Em relação ao WCST, uma metanálise (32 artigos) realizada por Romine e colaboradores (2004) apontou características de sensitividade e especificidade adequadas deste instrumento entre grupos com TDAH e grupos controle. Conforme esses autores, o tamanho do efeito (effect siz̨e) das variáveis do WCST variaram entre um efeito médio (0.5) (percentual de acertos, total de erros, número de categorias e erros perseverativos) e um baixo efeito (erros não-perseverativos e fracasso em manter o contexto). Tais dados suportam as evidências de outro estudo de metanálise, indicando em $70 \%$ dos estudos da avaliação de déficits do lobo frontal o uso do WCST (Alvarez \& Emory, 2006). Embora um pior desempenho no WCST possa indicar prejuízos no funcionamento do córtex frontal, esse resultado isolado não pode ser considerado suficiente para o diagnóstico de TDAH (Romine e colaboradores, 2004).

Em relação ao Stroop Test, o estudo de metanálise, realizado por Homack e Riccio (2004), apontou que diferentes versões desse instrumento têm sido utilizadas na avaliação de FE em crianças e adolescentes, sendo a versão de Charles Golden a mais comumente citada (em 22 dos 33 estudos revisados). De acordo com esses autores, o Stroop é capaz de discriminar prejuízos neuropsicológicos em crianças com TDAH quando comparadas a grupos não-clínicos. No entanto, não há consistência nos resultados para discriminar o grupo de crianças TDAH de outros grupos clínicos. Os resultados indicaram especificidade do Stroop para discriminar crianças com TDAH de crianças com transtorno de conduta, comportamento disruptivo e problemas emocionais; mas não foi capaz de discriminar crianças com problemas de aprendizagem, autismo e síndrome de Tourette. Nesse sentido, embora um pior desempenho no Stroop seja indicativo de prejuízos frontais, este não é suficiente para o diagnóstico de TDAH.

\section{Autismo}

Foram encontradas 26 pesquisas enfocando os aspectos neuropsicológicos do autismo. A maioria desses estudos $(n=10)$ apontou déficits em FE e na memória ( $\mathrm{n}=$ 6). De um modo geral, baixo desempenho no WCST (Shu, Lung, Tien \& Chen, 2001) e falhas nas habilidades sociais, na comunicação, na memória de trabalho, na atenção e no comportamento direcionado a metas foram relacionadas ao baixo desempenho nas FE (Bishop \& Norbury, 2005; Gilotty, Kenworthy, Sirian, Black \& Wagner, 2002; Ruble, $\&$ Scott, 2002).

Contudo, pesquisas têm apontado peculiaridades na disfunção executiva no autismo e entre os diferentes grupos de crianças com transtornos do desenvolvimento. Nesse sentido, um estudo investigou a presença de prejuízos em $\mathrm{FE}$ em crianças autistas e em crianças com TDAH, comparando-as ao grupo controle, enfocando a inibição de resposta, o planejamento, a flexibilidade cognitiva e a memória (working memory) (Goldberg e colaboradores, 2005). Os resultados indicaram que o grupo autista obteve pior desempenho na memória imediata, porém não foi encontrada diferença significativa entre os grupos nas demais tarefas. Déficits em tarefas verbais, que necessitavam de maior habilidade em mudança cognitiva, e na iniciativa para estratégias de recuperação léxica foram encontrados em jovens adultos com autismo e/ou transtorno de Asperger, enquanto a inibição de resposta não apresentou alteração (Kleinhans, Akshoomoff \& Delis, 2005). O estudo de Ozonoff e Strayer (2001) apontou que não houve diferença entre crianças com autismo e o grupo controle em tarefas neuropsicológicas que avaliavam working memory. Em contrapartida, prejuízos nessa função foram encontrados em crianças autistas, bem como inibição de controle e planejamento, embora estes não tenham sido correlacionados a déficits de linguagem (Joseph, McGrath \& Tager-Flusberg, 2005). Nesse sentido, é necessária certa cautela na afirmação de que FE estão afetas no autismo, uma vez que os prejuízos podem ser 
específicos e não globais. Ainda, ao avaliar a presença da diferença de desempenho em FE entre crianças autistas, crianças com transtorno de Asperger e crianças com transtorno do desenvolvimento não especificado, uma pesquisa observou que este último grupo apresentou melhor desempenho do que os dois primeiros (Verte, Geurts, Roeyers, Oosterlaan \& Sergeant, 2006).

Em relação à memória, um pior desempenho no funcionamento visual, verbal e espacial foi encontrado em crianças autistas (Williams, Goldstein \& Minshew, 2006). Mediante uma bateria neuropsicológica da memória e de ressonância magnética, foi encontrada uma associação entre os prejuízos dessa função e o lobo temporal medial (Salmond, Ashburner, Conelly, Friston, Gadian \& VerghaKhadem, 2005). Em oposição a este dado, crianças autistas não apresentaram falhas nem em tarefas de memória implícita nem de memória explícita, sugerindo que não há evidências de déficits no lobo temporal medial (Renner, Klinger \& Klinger, 2000). Ainda, crianças com diferentes níveis de autismo apresentaram desempenhos variados em tarefas de memória (Bebko \& Ricciuti, 2000) e usaram mais estratégia de recência na recuperação de conteúdo verbal (Renner e colaboradores, 2000).

\section{Transtornos de bumor}

$\mathrm{Na}$ literatura revisada, observou-se uma maior ênfase sobre a avaliação neuropsicológica do transtorno bipolar (TB) entre os transtornos de humor na infância e adolescência (11 de 15 estudos). De um modo geral, pacientes pediátricos com TB apresentaram prejuízos na memória declarativa, atenção sustentada, atenção visual, flexibilidade cognitiva e velocidade psicomotora (Dickstein e colaboradores, 2004; Doyle e colaboradores, 2005; Glahn e colaboradores, 2005). Pior desempenho em tarefa de cognição social e flexibilidade de resposta foram encontrados em um grupo pediátrico de TB (McClure e colaboradores, 2005). Ao revisarem estudos neuropsicológicos e de neuroimagem, Caetano e colaboradores (2005) indicaram déficits na atenção, memória e em FE em crianças e adolescentes com TB, quando comparados a grupo controle e outros grupos clínicos. Os estudos de neuroimagem sugeriram anormalidades no funcionamento do circuito frontallímbico associadas ao TB.

Pesquisas realizadas, ainda, propuseram que os déficits neuropsicológicos do TB tanto podem estar associados a comorbidade com o TDAH, para tarefa de atenção mapeada em fMRI scans (Adler e colaboradores, 2005), quanto podem ser independentes, para atenção sustentada, memória e velocidade de processamento (Doyle e colaboradores, 2005). Um estudo longitudinal indicou que $67 \%$ dos jovens adultos com TB apresentaram desempenho mais baixo no WCST, avaliados na adolescência, quando foram comparados a indivíduos sem depressão maior (17\%) e com depressão unipolar $(19 \%)$ (Meyer e colaboradores, 2004).

Além disso, diferenças no desempenho cognitivo entre crianças deprimidas e crianças ansiosas foram investigadas. Nesse sentido, crianças e adolescentes com depressão (grupo depressão) e ansiedade (grupo ansiedade) foram avaliadas mediante tarefas de atenção e de memória, e comparadas a grupo controle (Günther, Holtkamp, Jolles, Herpertz-Dahlmann \& Konrad, 2004). O resultado desse estudo apontou uma dissociação entre os grupos depressão e ansiedade, tendo sido encontrados prejuízos na memória verbal apenas em crianças deprimidas. Não foram observadas diferenças no desempenho da atenção entre esses dois grupos. Em outro estudo, meninos ansiosos e deprimidos apresentaram déficits na seqüência, na alternância e na resolução de problemas, evidenciando prejuízo no funcionamento do lobo frontal (Emerson, Mollet \& Harrison, 2005). Ainda crianças deprimidas obtiveram pior desempenho no Verbal Fluency Test e no Stroop Test do que o grupo controle, sugerindo problemas na atenção e em FE (Cataldo, Nobile, Lorusso, Battaglia \& Molteni, 2005).

\section{Transtorno de conduta}

Nesta revisão foram encontrados 12 estudos referentes aos prejuízos neuropsicológicos do transtorno de conduta (TC), transtorno desafiador opositivo (TDO) e/ou comportamento delinqüente. $\mathrm{O}$ TC na infância e a delinqüência juvenil têm sido relacionados à presença de déficits em FE (Kelly, Richardon, Hunter \& Knapp, 2002; Veneziano, Veneziano, LeGrand \& Richards, 2004). Foram encontradas três pesquisas de revisão teórica sobre a presença de prejuízos cognitivos relacionados ao TC (Hill, 2002; Raine, 2002; Teichner \& Golden, 2000). Tais pesquisas providenciam um suporte teórico acerca de déficits neuropsicológicos associados às $\mathrm{FE}$, particularmente, em relação a falhas na inibição do controle de impulso, na avaliação das conseqüências e na regulação do afeto. Da mesma forma, esses estudos indicaram a presença de outras variáveis (genéticas, temperamentais e ambientais) no desenvolvimento do TC, bem como sugeriram um continuum desses déficits ao longo do desenvolvimento.

Um outro aspecto apontado nos estudos sobre prejuízos neuropsicológicos do TC diz respeito à presença de comorbidade com o TDAH (Oosterlaan e colaboradores, 2005; Toupin, Déry, Pauzé, Mercier \& Fortin, 2000). No estudo longitudinal de Toupin e colaboradores (2000), crianças com TC, em follow-up de um ano, apresentaram pior desempenho em quatro de cinco tarefas de FE. Os sintomas de TDAH foram considerados preditores de TC, mas não o desempenho cognitivo. Já na 
pesquisa de Oosterlaan e colaboradores (2005) não foram encontrados déficits em FE, em crianças com TC. Porém a presença TDAH parece predizer um pior desempenho em $\mathrm{FE}$ nos casos de comorbidade entre TDAH/TDO/TC. Desta forma, os déficits nas FE envolvidos nos casos de TC ainda não estão totalmente esclarecidos.

\section{Considerações finais}

O presente estudo buscou apontar um panorama das publicações científicas recentes na área da avaliação neuropsicológica da criança e do adolescente. Como pode ser visto na sessão de resultados, os estudos que investigam aspectos neuropsicológicos dos transtornos na infância dedicam-se basicamente à avaliação das FE, sobretudo no que se refere aos prejuízos do córtex pré-frontal no TDAH, autismo, TB e TC. Em relação ao autismo, os resultados controversos impedem uma generalização dos déficits em FE. Crianças deprimidas apresentaram pior desempenho na memória e em FE, e nos casos de TB pediátrico foram encontrados prejuízos na memória, na atenção e em FE. Esses dados corroboram o estudo de Rocca e Lafer (2006), referente aos aspectos neuropsicológicos do TB. $\mathrm{Na}$ mesma direção, aspectos neuropsicológicos da depressão, incluindo alterações na atenção sustentada, no controle inibitório e na capacidade de alternância de foco atentivo foram apontados por Rozenthal, Lakc e Engelhard (2004). Em relação aos problemas de conduta na infância e adolescência, pesquisas futuras se fazem necessárias, por não haver maior clareza nos achados neuropsicológicos encontrados até $\mathrm{O}$ momento. A presença de prejuízos nas FE também tem sido encontrada com adultos com comportamento antisocial e psicopatia (Morgan \& Lilienfeld, 2000). Nesse sentido, pesquisas longitudinais podem fornecer elementos para uma maior compreensão sobre mudanças ou permanência dos déficits cognitivos na evolução do TC na infância para comportamento anti-social na vida adulta.

Tendo em vista a predominância de alterações funcionais das FE nos transtornos psicológicos revisados, ressalta-se a importância de uma maior compreensão dos aspectos neurodesenvolvimentais das FE na área da neuropsicologia infantil, uma vez que estas se desenvolvem até o final da adolescência. A avaliação neuropsicológica das FE na criança pode fornecer subsídios à prevenção e à intervenção clínica, bem como elementos para um modelo conceitual mais complexo sobre os quadros psicológicos, integrando os prejuízos cognitivos às alterações comportamentais e emocionais. Em relação aos instrumentos utilizados na avaliação das FE, destaca-se a importância de novos estudos sobre a validação e a normatização de testes neuropsicológicos, buscando apontar critérios de fidedignidade e validade apropriados ao contexto sociocultural, à amostra selecionada (clínica e não-clínica) e aos diferentes quadros psicopatológicos e/ou neurológicos.

Ressalta-se, ainda, que poucos estudos brasileiros foram encontrados nesta revisão. Uma hipótese é que a avaliação neuropsicológica ainda seja um campo de atuação recente e pouco explorado pelos psicólogos, apesar da aprovação da neuropsicologia como uma área de especialização, pelo Conselho Federal de Psicologia. Nesse sentido, destaca-se a importância deste campo de atuação profissional, que pode contribuir para um maior conhecimento sobre as relações entre prejuízos cognitivos e psicopatologia. Sugere-se, ainda, a realização de estudos longitudinais que possam contribuir para uma melhor compreensão dos prejuízos cognitivos e dos efeitos da cronificação ou remissão dos sintomas ao longo do desenvolvimento. Uma maior integração entre as pesquisas clínicas e os estudos de neuroimagem pode aumentar a validade dos resultados sobre prejuízos cognitivos associados a transtornos psicológicos na infância.

Algumas limitações foram encontradas nos estudos revisados. A presença de comorbidades psiquiátricas, o uso de medicação psicotrópica, o pequeno tamanho das amostras, amostras muito específicas e a utilização de instrumentos reduzidos são algumas das limitações que podem comprometer a generalização dos resultados. Dessa forma, observa-se que são necessários cuidados metodológicos, principalmente na composição das amostras e na escolha dos instrumentos, para que os estudos sobre prejuízos neuropsicológicos na infância sejam mais consistentes.

\section{Referências}

Adler, C. M., Delbello, M. P., Mills, N. P., Schmithorst, V., Holland, S. \& Strakowski, S. M. (2005). Comorbid $\mathrm{ADHD}$ is associated with altered patterns of neuronal activation in adolescents with disorder performing a simple attention task. Bipolar Disorders, 7(6), 577-588.

Alvarez, J. A. \& Emory, E. (2006). Executive function and the frontal lobe: A meta-analytic review. Neuropsychology Review, 16(1), 17-42.

Amaral, A. H. \& Guerreiro, M. M. (2001). Transtorno do déficit de atenção e hiperatividade: proposta de avaliação neuropsicológica para diagnóstico. Arquivos de Neuropsiquiatria, 59(4), 884-888.

Anderson, V. A., Anderson, P., Northam, E., Jacobs, R. \& Mikiewicz, O. (2002). Relationships between cognitive and behavioral measures of executive function in children with brain disease. Child Neuropsychology, 8(4), 231-240.

Bebko, J. M. \& Ricciuti, C. (2000). Executive functioning and memory strategy use in children with autism: The 
influence of task constraints on spontaneous rehearsal. Autism, 4(3), 299-320.

Bedard, A. C., Ickowicz, A. \& Tannock, R. (2002). Methylphenidate improves Stroop naming speed, but not response interference, in children with attention deficit hyperactivity disorder. Journal of Child and Adolescent Psychopharmacology, 12(4), 301-309.

Bedard, A. C., Martinussen, R., Ickowicz, A. \& Tannock, R. (2004). Methylphenidate improves visual-spational memory in children with attention-deficit/hyperactivity disorder. Journal of American Academy Child and Adolescent Psychiatry, 43(3), 260-268.

Beers, S. R. \& De Bellis, M. D. (2002). Neuropsychological function in children with maltreatment-related posttraumatic stress disorder. American Journal Psychiaty, 159 (3), 483-486.

Bishop, D. V. \& Norbury, C. F. (2005). Executive functions in children with communication impairments, in relation to autistic symptomatology. 2: Response inhibition. Autism, 9(1), 29-43.

Caetano, S. C., Olvera, R. L., Glahn, D., Fonseca, M., Pliszka, S. \& Soares, J. C. (2005). Fronto-limbic brain abnormalities in juvenile onset bipolar disorder. Biology Psychiatry, 58(7), 525-531.

Cataldo, M. G., Nobile, M., Lorusso, M. L., Battaglia, M. \& Molteni, M. (2005). Impulsivity in depressed children and adolescents: A comparison between behavioral and neuropsychological data. Psychiatry Research, 136(2-3), 123-133.

Cornoldi, C., Marzocchi, G. M., Belotti, M., Caroli, M. G., Meo, T. \& Braga, C. (2001). Working memory interference control deficit in children referred by teachers for ADHD symptoms. Neuropsychology, Development, and Cognition Section C Child Neuropsychology, 7(4), 230-240.

Dickstein, D. P., Treland, J. E., Snow, J. McClure, E. B., Mehta, M. S., Towbin, K. E., Pine, D. S. \& Leibenluft, E. (2004). Neuropsychological performance in pediatric bipolar disorder. Biology Psychiaty, 55(1), 32-39.

Donders, J. \& Nesbit-Greene, K. (2004). Preditors of neuropsychological test performance after pediatric traumatic brain injury. Assessment, 11(4), 275-284.

Doyle, A. E., Biederman, J., Seidman, L. J., Weber, W. \& Faraone, S. V. (2000). Diagnostic efficiency of neuropsychological test scores for discriminating boys with and without attention deficit-hyperactivity disorder. Journal of Consulting and Clinical Psychology, 68(3), 477-488.

Doyle, A. E., Wilens, T. E., Kwon, A., Seidman, L. J., Faraone, S. V., Fried, R., Swezey, A., Snyder, L. \&
Blederman, J. (2005). Neuropsychological functioning in young with bipolar disorder. Biological Psychiatry, 58(7), 540-548.

Drechsler, R., Brandeis, D., Foldenyi, M., Imhof, K. \& Steinhausen, H. C. (2005). The course of neuropsychological functions in children with attention deficit hyperactivity disorder from late childhood to early adolescence. Journal of Child Psychology and Psychiatry, 46(8), 824-836.

Emerson, C. S., Mollet, G. A. \& Harrison, D. W. (2005). Anxious-depression in boys: an evaluation of executive functioning. Archives of Clinical Neuropsychology, 20(4), 539546.

Fischer, M., Barkley, R. A., Smallish, L. \& Fletcher, K. (2005). Executive functioning in hyperactive children as young adults: attention, inhibition, response perseveration, and the impact of comorbidity. Developmental Neuropsychology, 27(1), 107-133.

Frazier, T. W., Demaree, H. A. \& Youngstrom, E. A. (2004). Meta-analysis of intellectual and neuropsychological test performance in attentiondeficit/hyperactivity disorder. Neuropsychology, 18(3), 543555.

Gilotty, L., Kenworthy, L., Sirian, L., Black, D. O. \& Wagner, A. F. (2002). Adaptive skills and executive function in autism spectrum disorders. Child Neuropsychology, 8(4), 241-248.

Glahn, D. C., Bearden, C. E., Caetano, S., Fonseca, M., Najt, P., Hunter, K., Pliszka, S. P., Olvera, R. L. \& Soares, J. C. (2005). Declarative memory impairment in pediatric bipolar disorder. Bipolar Disorders, 7(6), 546-554.

Goldberg, M. C., Mostofsky, S. H., Cutting, L. E., Mahone, E. M., Astor, B. C., Denckla, M. B. \& Landa, R. J. (2005). Subtle executive impairment in children with autism and children with ADHD. Journal of Autism \& Developmental Disorders, 35(3), 279-293.

Guimarães, I. E., Ciasca, S. M. \& Moura-Ribeiro, M. V. (2002). Neuropsychological evaluation of children after ischemic cerebrovascular disease. Arquivos de Neuropsiquiatria, 60(2b), 386-389.

Guimarães, C. A., Souza, E. A. P., Montenegro, M. A., Cendes, F. \& Guerreiro, M. M. (2003). Cirurgia para epilepsia na infância: avaliação neuropsicológica e de qualidade de vida. Arquivos de Neuropsiquiatria, 61(3b), 786-792.

Günther, T., Holtkamp, K., Jolles, J., Herpertz-Dahlmann, B. \& Konrad, K. (2004). Verbal memory and aspects of attentional control in children and adolescents with anxiety disorders or depressive disorders. Journal of 
Affective Disorders, 82(2), 265-269.

Hill, J. (2002). Biological, psychological and social processes in the conduct disorders. Journal of Child Psychology and Psychiatry, 43 (1), 133-164.

Hinshaw, S. P., Sam, N., Treuting, J. J., Carte, E. T. \& Zupan, B. A. (2002). Preadolescent girls with attentiondeficit/hyperactivity disorder: II. Neuropsychological performance in relation to subtypes and individual classification. Joumal of Consulting and Clinical Psychology, 70(5), 1099-1111.

Homack, S. \& Riccio, C. A. (2004). A meta-analysis of the sensitivity and specificity of the Stroop Color and Word Test with children. Archives of Clinical Neuropsychology, 19(6), 725-743.

Joseph, R. M., McGrath, L. M. \& Tager-Flusberg, H. (2005). Executive dysfunction and its relation to language ability in verbal school-age children with autism. Developmental Neuropsychology, 27(3), 361-378.

Kelly, T., Richardson, G., Hunter, R. \& Knapp, M. (2002). Attention and executive function deficits in adolescent sex offenders. Child Neuropsychology, 8(2), 138-143.

Kleinhans, N., Akshoomoff, N. \& Delis, D. C. (2005). Executive functions in autism and Asperger's disorder: Flexibility, fluency, and inhibition. Developmental Neuropsychology, 27(3), 379-401.

Konrad, K., Günther, T., Hanisch, C. \& HerpertzDahlmann, B. (2004). Differential effects of methylphenidate on attentional functions in children with attention-deficit/hyperactivity disorder. Journal of American Academy Child and Adolescent Psychiatry, 43(2), 191-198.

Koschack, J., Kunert, H. J., Derichs, G., Weniger, G. \& Irle, E. (2003). Impaired and enhanced attentional function in children with attention deficit/hyperactivity disorder. Psychological Medicine, 33(3), 481-489.

Lezak, M. D., Howieson, D. B. \& Loring, D. W. (2004). Neuropsychological assessment. (4th ed.). New York: Oxford University Press.

Lindsay, R. L., Tomazic, T., Levine, M. D. \& Accardo, P. J. (2001). Attentional function as measured by a Continuous Performance Task in children with dyscalculia. Journal of Developmental and Behavioral Pediatrics, 22(5), 287-292.

McClure, E. B., Treland, J. E., Snow, J., Schmajuk, M., Dickstein, D. P., Towbin, K. E., Charney, D. S., Pine, D. S. \& Leibenluft, E. (2005). Deficits in social cognition and response flexibility in pediatric bipolar disorder. American Journal Psychiatry, 162(9), 1644-1651.

Meyer, S. E., Carlson, G., A., Wiggs, E. A., Martinez, P. E.,
Ronsaville, D. S., Klimes-Dougan, B., Gold, P. \& Radke-Yarrow, M. (2004). A prospective study of the association among impaired executive functioning, childhood attentional problems, and the development of bipolar disorder. Development and Psychopathology, 16(2), 461-476.

Morgan, A. B. \& Lilienfeld, S. O. (2000). A meta-analytic review of the relation between antisocial behavior and neuropsychological measures of executive function. Clinical Psychology Review, 20(1), 113-136.

Nigg, J. T., Blaskey, L. G., Huang-Pollock, C. L. \& Rappley, M. D. (2002). Neuropsychological executive functions and DSM-IV ADHD subtypes. Journal of American Academy Child and Adolescent Psycbiatry, 41(1), 59-66.

O’Driscoll, G. A., Dépatie, L., Holahan, A-L., SavionLemleux, T., Barr, R.G., Jolicoeur, C. \& Douglas, V. I. (2005). Executive functions and methylphenidate response in subtypes of attention-deficit/hyperactivity disorder. Biology Psychiaty, 57(11), 1452-1460.

Oosterlaan, J., Scheres, A. \& Sergeant, J. A. (2005). Which executive functioning are associated with $\mathrm{AD} / \mathrm{HD}$, $\mathrm{ODD} / \mathrm{CD}$ and comorbid ADHD+ODD/CD? Journal of Abnormal Child Psychology, 33(17), 69-85.

Ozonoff, S. \& Strayer, D. L. (2001). Further evidence of intact working memory in autism. Journal of Autism \& Developmental Disorders, 31(3), 257-263.

Papadopoulos, T. C., Panayiotou, G., Spanoudis, G. \& Natsopoulos, D. (2005). Journal of Abnormal Child Psychology, 33(5), 611-623.

Perugini, E. M., Harvey, E. A., Lovejoy, D. W., Sandstrom, K. \& Webb, A. H. (2000). The predictive power of combined neuropsychological measures for attentiondeficit/hyperactivity disorder in children. Neuropsychology, Development, and Cognition Section C Cbild Neuropsychogy, 6(2), 101-114.

Raine, A. (2002). Annotation: The role of prefrontal deficits, low autonomic arousal, and early health factors in the development of antisocial and aggressive behavior in children. Journal of Child Psychology and Psychiatry, 43(4), 417-434.

Reeve, W. V. \& Schandler, S. L. (2001). Frontal lobe functioning in adolescents with attention deficit hyperactivity disorder. Adolescence, 36(144), 749-765.

Renner, P., Klinger, L. G. \& Klinger, M. R. (2000). Implicit and explicit memory in autism: Is autism an amnesic disorder? Journal of Autism \& Developmental Disorders, 30(1), 3-14.

Rocca, C. C. A. \& Lafer, B. (2006). Alterações neuropsicológicas no transtorno bipolar. Revista Brasileira 
de Psiquiatria, 28(3), 226-237.

Romine, C. B., Lee, D., Wolfe, M. E., Homack, S., George, C. \& Riccio, C. A. (2004). Wisconsin Card Sorting Test with children: a meta-analytic study of sensitivity and specificity. Archives of Clinical Neuropsychology, 19(8), 10271041.

Roth, R. M., Milovan, D., Baribeau, J. \& O'Connor, K. (2005). Neuropsychological functioning in early- and late-onset obsessive-compulsive disorder. Journal of Neuropsychiatry and Clinical Neurosciences, 17(2), 208-213.

Rozenthal, M., Laks, J. \& Engelhardt, E. (2004). Aspectos neuropsicológicos da depressão. Revista de Psiquiatria do Rio Grande do Sul, 26(2), 204-212.

Rubia, K., Taylor, E., Smith, A. B., Oksanen, H., Overmeyer, S. \& Newman, S. (2001). Neuropsychological analyses of impulsiveness in childhood hyperactivity. British Journal of Psychiatry, 179, 138-143.

Ruble, L. A. \& Scott, M. M. (2002). Executive functions and the natural habitat behaviors of children with autism. Autism, 6(4), 365-381.

Salmond, C. H., Ashburner, J., Cornelly, A., Friston, K. J., Gadian, D. G. \& Vargha-Khadem, F. (2005). The role of medial temporal lobe in autistic spectrum disorder. The European Journal of Neuroscience, 22(3), 764-772.

Sarkis, S. M., Sarkis, E. H., Marshall, D. \& Archer, J. (2005). Self-regulation and inhibition in comorbid ADHD children: An evaluation of executive functions. Journal of Attention Disorder, 8(3), 96-108.

Schmitz, M., Cadore, L., Paczko, M., Kipper, L., Chaves, M., Rohde, L. A., Moura, C. \& Knijnik, M. (2002). Neuropsychological performance in DSM-IV ADHD subtypes: an exploratory study with untreated adolescents. Canadian Journal of Psychiatry, 47(9), 863-869.

Schulz, K. P., Tang, C. Y., Fan, J., Marks, D. J., Newcom, J. H., Cheung, A. M. \& Halperin, J. M., (2005). Differential prefrontal cortex activation during inhibitory control in adolescents with and without childhood attentiondeficit/hyperactivity disorder. Neuropsychology, 19(3), 390402.

Seidman, L. J., Biederman, J., Monuteaux, M. C., Valera, E., Doyle, A. E. \& Faraone, S. V. (2005). Impact of gender and age on executive functioning: do girls and boys with and without attention deficit hyperactivity disorder differ neuropsychologically in preteen and teenage years? Developmental Neuropsychology, 27(1), 79-105.
Shallice, T., Marzocchi, G. M., Coser, S., Del Savio, M., Meuter, R. F. \& Rumiati, R. I. (2002). Executive function profile of children with attention deficit hyperactivity disorder. Developmental Neuropsychology, 21(1), 43-71.

Shu, B. C., Lung, F. W., Tien, A. Y. \& Chen, B. C. (2001). Executive function deficits in non-retarded autistic children. Autism, 5(2), 165-174.

Steinlin, M., Imfeld, S., Zulauf, P., Boltshauser, E., Lövblad, K. O., Lüthy, A. R., Perrig, W. \& Kaufmann, F. (2003). Neuropsychological long-term sequelae after posterior fossa tumour resection during childhood. Brain, $126(\mathrm{Pt}$ 9), 1998-2008.

Teichner, G. \& Golden, C. J. (2000). The relationship of neuropsychological impairment to conduct disorder in adolescence: a conceptual review. Aggression and Violent Behavior, 5(6), 509-528.

Toren, P., Sadeh, M., Wolmer, L., Eldar, S., Koren, S., Weizman, R. \& Laor, N. (2000). Neurocognitive correlates of anxiety disorders in children: a preliminary report. Journal of Anxiety Disorders, 14(3), 239-247.

Toupin, J., Déry, M., Pauzé, R., Mercier, H. \& Fortin, L. (2000). Cognitive and familial contributions to conduct disorder in children. Journal of Child Psychology and Psychiatry, 41(3), 333-344.

Veneziano, C., Veneziano, L., LeGrand, S. \& Richards, L. (2004). Neuropsychological executive functions of adolescents sex offenders and nonsex offenders. Perceptual and Motor Skills, 98(2), 661-674.

Verte, S., Geurts, H. M., Roeyers, H., Oosterlaan, J. \& Sergeant, J. (2006). Executive functioning in children with an autism spectrum disorder: Can we differentiate within the spectrum? Journal of Autism \& Developmental Disorders, 36(3),351-372.

Westerberg, H., Hirvikoski, T., Forssberg, H. \& Klingberg, T. (2004). Visuo-spatial working memory span: a sensitive measure of cognitive deficits in children with ADHD. Cbild Neuropsychology, 10(3), 155-161.

Williams, D. L., Goldstein, G. \& Minshew, N. J. (2006). The profile of memory function in children with autism. Neuropsychology, 20(1), 21-29.

Recebido em novembro de 2006 Reformulado em abril de 2007 Aprovado em maio de 2007 
Sobre as autoras:

Jeane Lessinger Borges é psicóloga, graduada pela Universidade do Vale do Rio dos Sinos (UNISINOS), aluna de mestrado do Programa de Pós-Graduação em Psicologia da Universidade Federal do Rio Grande do Sul (UFRGS) e do Curso de Especialização em Neuropsicologia (UFRGS).

Clarissa Marceli Trentini é psicóloga, especialista em Psicologia Clínica: ênfase em Avaliação Psicológica pela Universidade Federal do Rio Grande do Sul (UFRGS), mestre em Psicologia Clínica pela Pontifícia Universidade Católica do Rio Grande do Sul (PUCRS) e Doutora em Ciências Médicas - ênfase em Psiquiatria (UFRGS). Atualmente é professora adjunta do Instituto de Psicologia (UFRGS).

Denise Ruschel Bandeira é psicóloga, mestre e doutora em Psicologia do Desenvolvimento (UFRGS), professora e coordenadora do Programa de Pós-Graduação em Psicologia da Universidade Federal do Rio Grande do Sul (UFRGS).

Débora Dalbosco Dell'Aglio é psicóloga, mestre e doutora em Psicologia do Desenvolvimento (UFRGS), professora do Programa de Pós-Graduação em Psicologia da Universidade Federal do Rio Grande do Sul (UFRGS) e chefe do Departamento de Psicologia do Desenvolvimento do Instituto de Psicologia (UFRGS). 
